\title{
Seeing CCR5
}

\section{By Tracey Baas, Senior Editor}

Despite offering a new mechanism for inhibiting HIV, Selzentry maraviroc's sales have languished. Pfizer Inc.'s CC chemokine receptor 5 antagonist does not block viruses with tropism for another co-receptor, CXC chemokine receptor 4 , and many physicians find tropism assays too costly and too slow.

Now, a Shanghai and La Jolla team has reported the crystal structure of human CC chemokine receptor 5 (CCR5; CD195) bound to Selzentry, which provides a better understanding of how HIV

\section{"The team made slight} changes to the receptor in order to be able to characterize its structure but in doing so might have made substantial functional alterations. Tiny changes in the structure of ligands for this type of receptor can produce large changes in protein functional character that cannot be predicted and will affect how one goes about designing therapeutics."

-Tony Wood, Pfizer Inc. virions interact with co-receptors before gaining cellular entry. ${ }^{1}$ The structural snapshot could be used to guide discovery of better versions of viral entry inhibitors.

Maraviroc is marketed by Pfizer in collaboration with GlaxoSmithKline plc for patients with CCR5-tropic HIV-1 infection. The small molecule posted combined sales of $£ 96$ million (\$126.5 million) in 2012.

HIV gains entry into immune cells when HIV gp120 binds to the cellular receptor CD4 and either of its co-receptors, CXC chemokine receptor 4 (CXCR4; NPY3R) or CCR5. Binding of HIV to CD4 and one of the co-receptors induces structural rearrangements in the gp120-containing trimeric virus envelope complexes, ultimately resulting in fusion of the virus with the cellular membrane and subsequent viral entry.

Most HIV infections occur initially via CCR5, but the virus often evolves to switch co-receptor usage from CCR5 to CXCR4, opening up more cell types to virus infection. This evolution also can lead to resistance against Selzentry.

Obtaining structural information for CCR5 and CXCR4 had been challenging because the two class A GPCRs are so structurally dynamic that they move easily through a range of conformations between active and inactive signaling states, making them difficult to crystallize.

In 2010, a team from The Scripps Research Institute led by professor of molecular biology Raymond Stevens was finally able to get CXCR4 crystals using stabilizing approaches, but CCR5 crystals remained elusive because of a host of technical challenges. ${ }^{2}$
The CXCR4 structure helped guide drug discovery and lead optimization. CXCR4 structure-based virtual ligand screening studies exhibited hit rates of $20 \%-70 \%{ }^{3-6}$

Now, a member of Stevens' original team has used what she learned from the CXCR4 study to tackle CCR5. Beili Wu, a professor at the Shanghai Institute of Materia Medica (SIMM) of the Chinese Academy of Sciences, led her own team and determined the structure of CCR5. The new team included researchers from SIMM and the Scripps Research Institute, including Stevens.

The first step was making the target less dynamic. Thus, the team engineered a human CCR5 construct with multiple modifications. These included taking out 33 amino acids from the C-terminus to increase thermostability, substituting 3 amino acids to increase both general stability and detergent solubility and substituting one more amino acid to help keep the protein in its inactive state.

To further stabilize the protein's inactive conformation, they focused on crystallizing CCR5 complexed with Selzentry.

The team determined the structure of the complex at $2.7 \AA$ resolution and showed a number of similarities to CXCR4, such as the seven transmembrane $\alpha$-helices bundling in a similar fashion.

One of the major differences was how the allosteric inverse agonist Selzentry interacted with CCR5 versus how a competitive antagonist, an isothiourea derivative, interacted with CXCR4. Selzentry penetrated more deeply into the CCR5 binding pocket than the competitive antagonist did within the CXCR4 binding pocket. Also, the CCR5 binding pocket was more open than the CXCR4 binding pocket.

Selzentry occupied the bottom of a deep binding pocket surrounded by helices, and the drug's phenyl group reached further down to form hydrophobic interactions with five aromatic residues. The triazole, tropane and cyclohexane moieties of the drug also fit into small subpockets to make individual hydrophobic contacts within the receptor.

These data further confirmed that Selzentry works by locking the receptor structure into an HIV-insensitive conformation rather than by physically blocking the virus from interacting with the receptor.

Results were published in Science.

"The CCR5-Selzentry structure reveals a chemokine binding site quite different from the CXCR4 structure. The CCR5 structure is thus a valuable new and different template for the homology modeling of potentially many other important receptors," said Jon Mason, senior research fellow at GPCR drug discovery company Heptares Therapeutics Ltd.

Per Johan Klasse, an associate professor of molecular biology and immunology at Weill Cornell Medical College, thinks that the CCR5Selzentry complex structure will enable approaches to improve drug design. "The exact contacts of maraviroc with the receptor can be modified to increase drug potency and also alter the conformational effect of maraviroc binding, making it harder for the virus to overcome the block," he said.

"It might also be useful to use a peptide that represents the third variable region loop of HIV gp120-the binding portion of gp120-instead of inhibitors and solve the structure of CCR5 or CXCR4 with those. Then they could be compared with the inhibited forms," added Klasse.

This would provide multiple snapshots of the receptors and help 
create molecular dynamic simulations that could better guide drug development.

\section{More snapshots}

Wu's team will next undertake structural studies of CCR5 and CXCR4 in complex with HIV gp120 and CD4 to obtain more informative pictures of the process of viral infection.

"The structures of complexes between the co-receptors and gp120-CD4 are needed to fully understand mechanisms of HIV-1 infection. The structural information will help to identify key interactions between gp120 and the co-receptors, which will facilitate the development of new drugs that block the specific interactions," she said.

Sam Williams, CEO of C4X Discovery Ltd., said that dynamic modeling should be the next step. "X-ray co-crystallography can only provide a static picture of what a ligand looks like when bound to its target. Understanding the behavior of a ligand in solution in the unbound state demonstrates what transition the ligand has to make to bind the target and provides a fuller understanding of the relative binding affinities of different ligands from the same class," he said.

"Using NMR-based methods for accurate determination of ligandsolution structures would help to provide this dynamic picture and reveal novel routes forward for ligand optimization," added Thorsten Nowak, senior medicinal chemist at $\mathrm{C} 4 \mathrm{X}$.

Tony Wood, SVP of medicinal chemistry at Pfizer and discoverer of Selzentry, agreed that molecular dynamics would provide a richer understanding of fundamental interactions between drug and receptor with which to guide drug research.

He also said that it is difficult to know if the modification used to stabilize the human CCR5 construct made substantial changes to the character of CCR5 without more functional evaluation. "The team made slight changes to the receptor in order to be able to characterize its structure but in doing so might have made substantial functional alterations," he said. "Tiny changes in the structure of ligands for this type of receptor can produce large changes in protein character that cannot be predicted and will affect how one goes about designing therapeutics."

"Another more complex question that is not entirely clear is does the field need another CCR5 agonist?" asked Wood. "If yes, CCR5-targeting therapeutics have to be developed with caution so that they are safe from a cardiovascular and hepatic point of view."

Selzentry's label includes a black box warning about hepatotoxicity, as well as warning language about an increased risk of cardiovascular events.

Nevertheless, the SIMM team already has performed structure-based drug design and has obtained several lead compounds with more potent antiviral effects than Selzentry.

The team's CCR5 structure-based drug discovery project includes four SIMMs groups. Professor Hong Liu is leading compound synthesis, Deputy Director and Professor Hualiang Jiang is leading drug design, Professor Xin Xie is leading functional assays and $\mathrm{Wu}$ will continue to lead structural biology. Each scientist also played a role in the CCR5 structure project.

The findings from the study are not patented.

Other CCR5 inhibitors in development include CytoDyn Inc.'s PRO 140, which is in Phase II trials, and Tobira Therapeutics Inc.'s cenicriviroc, a dual CCR5 and CCR2 (CD192) antagonist, which is in Phase II development. Tobira plans to advance the compound to Phase III at the end of 2013.

Baas, T. SciBX 6(39); doi:10.1038/scibx.2013.1086

Published online Oct. 10, 2013

\section{REFERENCES}

1. Tan, Q. et al. Science; published online Sept. 12, 2013; doi:10.1126/science.1241475

Contact: Beili Wu, Shanghai Institute of Materia Medica, Chinese Academy of Sciences, Shanghai, China e-mail: beiliwu@simm.ac.cn

2. Wu, B. et al. Science 330, 1066-1071 (2010)

3. Shoichet, B.K. \& Kobilka, B.K. Trends Pharmacol. Sci. 33, 268-272 (2012)

4. Mysinger, M.M. et al. Proc. Natl. Acad. Sci. USA 109, 5517-5522 (2012)

5. Mooring, S.R. et al. ChemMedChem 8, 622-632 (2013)

6. Planesas, J.M. et al. J. Mol. Graph. Model. 38, 123-136 (2012)

\section{COMPANIES AND INSTITUTIONS MENTIONED}

C4X Discovery Ltd., Manchester, U.K.

Chinese Academy of Sciences, Shanghai, China

CytoDyn Inc. (OTCBB:CYDY), Lutz, Fla.

GlaxoSmithKline plc (LSE:GSK; NYSE:GSK), London, U.K. Heptares Therapeutics Ltd., Welwyn Garden City, U.K. Pfizer Inc. (NYSE:PFE), New York, N.Y.

The Scripps Research Institute, La Jolla, Calif.

Shanghai Institute of Materia Medica, Shanghai, China

Tobira Therapeutics Inc., San Francisco, Calif.

Weill Cornell Medical College, New York, N.Y. 